\title{
Crues des cours d'eau du versant oriental des Cévennes en septembre-octobre 1958
}

Synthèse des faits ou des remarques retenues à la suite de la réunion des sections du 16 janvier 1959 et lues à l'ouverture de la session du 20 mars 1959

\section{Floods in watercourses on the Eastern slopes of the Cevennes, in September and October 1958}

Survey of facts and remarks taken into consideration as a result of the Section meeting on January 16th 1959 and presented at the opening of the session on March 20th 1959

DAR A. DE ROUVILLE

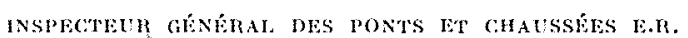

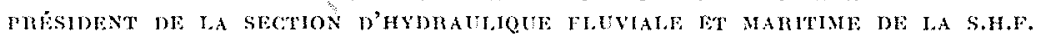

$1^{\circ}$ Il ne faut pas considérer qu'il existe un seul type d'inondations cévenoles, ni se laisser obnubiler par celles de septembre-octobre 1958 , dont le cycle, pour chacune, a été de l'ordre de vingt-quatre heures.

Il y a eu, dans le passé, des inondations causées par des pluies quasi-continues de trois ou quatre jours, se terminant quelquefois par une dernière journée de pluies diluviennes, qui ont achevé de transformer l'inondation en désastre; d'autres fois (comme en 1958), tout le drame s'est passé en une seule journée, avec plusieurs heures de pluies diluviennes, lotalisant quelques centaines de $\mathrm{mm}$ d'ean en vingt-quatre heures.

$2^{\circ}$ Plusieurs de ces catastrophes ont été doubles ou groupées, une première inondation ayant été suivie, après quelques jours d'accalmie, par une seconde; tel fut le cas des pluies des 29 el 30 septembre, d'une part, des $3-4$ octobre, d'autre part.

C'est à rapprocher du groupage de dépressions océaniques passant en «familles sur l'Europe occidentale.

$3^{\circ}$ Au point de vue météorologique, des vents de S.E., forts ou faibles, accompagnent nécessairement, ou produisent des pluies. Ils ont été très forts dans la matinée du 30 septembre, abattant des branches d'arbres encore feuillus, et même des arbres entiers, avant même les pluies, ce qui explique que celles-ci ont trouvé des aliments pour constituer des barrages devant les ponts et nourrir les fronts d'eau en progression rapide.

C'est cette violence qui, le 30 septembre, a été une cause aggravante supplémentaire des effets des inondations.

Mais, bien souvent, dans le passé, les vents de S.E. ont été faibles ou modérés.

4' L'Office National Météorologique a nettement mis en évidence l'existence d'une couche d'air froid de l'ouest ou du nord-ouest, régnant au-dessus des vents de S.E., couche qui, en rafraichissant les masses d'air humide d'origine méridionale, ou peut-être africaine, a précipité leur condensation en chutes impressionnantes.

Il serait intéressant de rechercher si une superposition semblable a accompagné, comme il est probable, les précédentes inondations.

On se rend compte maintenant que celles-ci sont rendues possibles, sinon fatales, par la coexistence de basses pressions dans l'ouest ou le nord-ouest (dans l'espèce du 30 septembre, le 
centre dépressionnaire était sur les Iles Britanniques) et de hautes pressions dans l'est, s'étendant assez avant dans le sud (jusqu'en Italie dans l'espèce du 30 septembre).

Cette double influence peut déterminer, en effet, des vents de S.E. très violents.

$5^{\circ}$ Il ne faut pas oublier cependant l'effet supplémentaire de la muraille de montagnes qui arrête les couches humides, les refroidit par ellemème et les fait précipiter en pluies violentes.

$6^{\circ}$ Une carte, qui fut projetée lors de la réunion, a montré très nettement l'avancée du front froid au-dessus des masses chaudes, en accusant les heures du début des pluies intenses, en progression lente du N.O. vers le S.E.

$7^{\circ}$ Il est remarqué que les postes d'observation de l'Óffice National Météorologique n'étaient pas assez denses pour enregistrer les phénomènes malheureusement constatés à terre, à l'exception d'un pluviomètre de Montélimar, qui a donné $180 \mathrm{~mm}$ en vingt-quatre heures.

Dans les hautes vallées des Cévennes, en effet, il n'a pas été indiqué de pluies exceptionnelles ni de vents du S.E. tries violents.

Cela indique, pour les phénomènes locaux ou régionaux, une insuffisance de notre réseau d'observations.

Quelques autres pluviometres non enregistrès sur le bulletin quotidien de l'O.N.M. ont indiqué cependant des chiffres compris entre 300 et $400 \mathrm{~mm}$ et au-delá; mais plusieurs d'entre eux n'ont pas été surveillés par les observateurs et sont restés limités à $180 \mathrm{~mm}$, chiffre approximatif de remplissage du pluviometre minimum ordinaire.

Il faudrait, ou de plus grands pluviometres, ou des pluviomètres enregistreurs (1), on un personnel osant sortir sous les abats d'eau au moment où le pluviomètre est supposé plein.

$8^{\circ}$ II y a eu encore trop d'incidents avec les pluviomètres, de même qu'avec les limnimètres, dont l'implantation dans des lits torrentiels reste délicate.

$9^{\circ}$ Il y a lieu de faire ressortir que ce sont les fortes pluies du 29 septembre dans les hautes vallées, et non pas celles du 30 dans les vallées moyennes, qui ont déterminé la première vague d'eau, à laquelle les suivantes se sont adjointes, l'ensemble expliquant l'espèce de mascaret qui a ravagé notamment la plaine du Gardon.

Et même la pluie a commencé dès le 28 septembre à 22 heures sur l'Aigoual. Mais ce n'est pas sur les hauts sommets et sur les crêtes qu'il

(1) M. PArné a émis le vou que 50 ombrographes soient installés entre les Cévennes et le Rhône. faut chercher les chutes les plus intenses. En l'espèce, le dòme des isohyètes (ligne d'égales pluies) était distant de quelques kilomètres de la ligne de partage des eaux, tout en lui étant parallèle (ce qui prouve néammoins l'influence de la haute montagne).

Les fortes pluies n'ont commencé, dans les moyennes vallées et dans les hautes Cévennes, que dans l'après-midi du 30 , très peu avant l'arrivée de la crue causée surtout, comme il est dit ci-dessus, par les pluies du 29.

Il faut admettre dans les montagnes la possibilité de pluies de l'ordre de $100 \mathrm{~mm}$ à l'heure (on dit avoir enregistré $135 \mathrm{~mm}$ à Montpellier pendant un quart d'heure seulement, tandis que les pluies des Cévennes paraisent pouvoir tomber avec cette intensité plusieur's heures de suite).

$10^{\circ}$ Il ne faut pas trop parler des orages; les phénomènes électriques ont été courts et n'ont pas été déterminants dans l'espèce.

11" Heureusement, on aura dans quelque temps plus de résultats pour les vallées, grâce au Centre d'Etudes de l'E.D.F., qui va relever l'O.N.M., notamment sur le Chassézac et sur le Vidourle, rivières qui ont donné lieu à des monographies particulières dans la présente réunion.

Toutefois, les observations méthodiques par l'E.D.F., si précieuses qu'elles soient pour l'avenir, sont encore trop récentes (1956) pour fournir tous les enseignements possibles.

Il en découle cependant déjà que, même dans un bassin karstique comme celui du Vidourle, le coefficient de ruissellement demeure inférieur à $60 \%$.

$12^{\circ}$ Il y a eu très vraisemblablement convergence d'ondes, aussi bien dans les bassins des Gardons avec ceux de Saint-Jean, de Mialet et d'Alès, que dans les hassins du Chassézac avec l'Altier, la Borne et le Chassézac proprement dit, qui convergent au même point.

$13^{\circ}$ Il y a plus d'incertitude sur les débits que sur les chutes d'eau, et si on peut espérer faire mieux à l'avenir, nòtamment srâce à l'E.D.F. qui s'intéresse à l'aménagement — depuis peu d'années il est rrai — de certaines rivières, on ne peut être trop affirmatif sur les chiffres des débits, ni se baser sur des formules reliant les déhits avec les hauteurs de crues et les chutes d'eau, d'autant moins que les lits des cour's d'eau ont pu s'exhausser on s'abaisser d'une crue à l'autre.

C'est ainsi qu'on a pu douter du débit de la Cèze dans le Rhòne à son embouchure, alors que le débit à l'amont était triple peut-être de celui que les échelles du Rhône ont accusé; mais on peut se demander si ce n'est pas le Rhône qui a refoulé les eaux de la Cèze en aggravant les 
inondations par cette rivière, ou le simple étalement de la rivière dans la plaine précédant son embouchure. M. Pardé a également des doutes sur la cote réellement atteinte par le Gardon en amont du pont de Ners.

$14^{\circ}$ Sont à retenir une montée de l'Hérault à Ganges, de $5,20 \mathrm{~m}$ en 45 minutes, une montée de $9 \mathrm{~m}$ du Chassézac, une montée de $100 \mathrm{~mm}$ par minute sur le Vidourle à Q'uissac, avec une célérité de $15 \mathrm{~km} / \mathrm{h}$ pour l'onde et une vitesse superficielle de $10 \mathrm{~m}$ à la seconde.

La vague qui a balayé la plaine a été évaluée par M. Pardé, qui n'en était pas témoin, à $80 \mathrm{~cm}$; il est probable qu'elle était plus élevée, sans aller jusqu'aux 2 ou 3 mètres relatés dans la Presse, car cela n'expliquerait pas le renversement des voitures légères et les ripages des poids lourds sur la route $\mathrm{n}^{\circ} 106$ de Nîmes à Ales; il n'est pas exclu que la vague ait eu $1,50 \mathrm{~m}$ environ de hauteur.

$15^{\circ}$ Malgré tous les modes récents de communication qui devraient être plus efficaces, semble-t-il, que naguère, maints intéressés paraissent avoir ignoré dans la plaine, vers SaintAmbroix ou Nozières, qu'il pleuvait à verse depuis la veille en amont de Bessèges ou de SaintJean-du-Gard.

Il y a là un systeme d'information insuffisant et qui doit être rénové.

16 "Toutes les études ont montré que, pour chacue rivière, il y avait eu une crue plus forte dans le passe que celles de 1958 (sauf peut-être pour la Ceze en aval de Saint-Ambroix); mais M. Pardé a fait ressortir que, si l'on considere l'ensemble des bassins et la quantité d'eau totale qu'ils ont dù recevoir, c'est peut-être un des plus forts cataclysmes de cette nature qu'ait subi le Languedoc.

Toutefois, pour chacun des sinistrés, e'est la crue de sa rivière qui l'intéresse et le frappe. $17^{\circ}$ Il n'y a pas eu, d'après M. Pardé qui a visité les lieux quelques semaines après, d'érosions comparables à celles de la vallée du Tech en 1940, où d'immenses étendues de flancs de montagnes ont été mises à nu.

Il n'y a pas eu davantage de désastre comparable à ceux de la Garonne en 1950, où l'on a pu dénombrer 3000 maisons détruites.

$18^{\circ}$ La brousse est au moins aussi efficace que la futaie pour atténuer l'effet des crues et le ravinement du sol; les feuillus tels que les hêtres sont plus efficaces que les conifères.

19. Il faut éviter de laisser multiplier les constructions, notamment les constructions industrielles, dans le champ des inondations, ni de laisser réduire la section d'écoulement des rivières par les ponts ou dans la traversée des villes (comme à Sommières, dont le pont, en partie aveuglé par le développement de la ville, a réduit de moitić la largeur du lit mineur).

M. Pardé attribue une influence à l'érosion des lits sous les ponts et à la formation corrélative d'un banc en aval des ponts, ce qui diminue la section d'écoulement.

La défense par digues longitudinales, envisagée parfois, est une arme à double tranchant, en ce sens qu'établie sur un plan trop étroit, elle relèverait le niveau des crues a l'aval, et si on écarte les digues suffisamment pour éviter ce danger, elles deviennent en général inefficaces pour la plupart des constructions imprudemment établies dans le champ d'inondation.

$20^{\circ}$ Il serait désirable de posséder des profils en travers successifs des rivieres et des plans pour les champs d'inondation.

Cela faciliterait notamment les consignes pour l'arrêt de la circulation sur les routes, qui doit etre impératif le moment venu et non indiqué sous forme de conseil. 